ORIENTAL JOURNAL OF CHEMISTRY

An International Open Free Access, Peer Reviewed Research Journal

www.orientjchem.org
ISSN: 0970-020 X

CODEN: OJCHEG

2014, Vol. 30, No. (4):

Pg. 2005-2009

\title{
Synthesis and Characterization of the Thiazolidinone and/or Thiourea substituted Amine Complexes of Cu (II)
}

\author{
ARCHANA SAXENA ${ }^{1 *}$ and RAJKUMAR UPADHYAY ${ }^{2}$ \\ 'Department of Chemistry, Swami Keshvanand Institute of Technology, \\ Management \& Gramothan, Jaipur - 302025, India. \\ 2Department of Chemistry, N.R.E.C College, Khurja - 203131, India. \\ ${ }^{*}$ Correspondnig author E-mail: draschem @gmail.com \\ http://dx.doi.org/10.13005/ojc/300464
}

(Received: September 09, 2014; Accepted: October 16, 2014)

\begin{abstract}
Mixed ligand complexes of copper (II), involving either Mercaptyl thiazolidinone (1) [2-(2hydroxy benzoyl)-3-N-(2-mercaptyl)-1-Thiazolidin-4-one] or Pyridine thiazolidinone (2) [ 2-(2hydroxy benzoyl-3-N-(2-Pyridinyl)-1-Thiazolidin-4-one] alone and along with Thiourea were synthesized by partial or complete replacement of the strongly coordinated ammonia of ammine complex. All the products have been characterized by elemental analysis, molar conductance, magnetic susceptibility, IR and electronic spectral measurements for their bonding and structures.
\end{abstract}

Key words: Mixed ligand complexes, Mercaptyl thiazolidinone, Pyridine thiazolidinone, Thiourea, Ammine complexes

\section{INTRODUCTION}

Thiazolidinones are the derivatives of thiazolidine which belong to an important group of heterocyclic compounds containing sulphur and nitrogen in a five member ring. The nucleus is also known as wonder nucleus because it gives out different derivatives with all different types of biological activities.

Chemistry of complexes derived by partial or complete replacement of strongly coordinated ammonia, halogen, cyanide or thiocyanate by organic ligands like Schiff's bases have rare mention ${ }^{[1-3]}$ in literature. Products obtained by replacement of ammonia of ammine complexes of copper(II), with 2-(2-hydroxy benzoyl)-3-N-(2mercaptyl)-1-Thiazolidin-4-one (1) or 2-(2-hydroxy benzoyl)-3-N-(2-Pyridinyl)-1-Thiazolidin-4-one(2) alone and along with thiourea have not been described hitherto and we now report their synthesis. Product(s), isolated as binary mixture(s) were resolved by column chromatography. 


\section{EXPERIMENTAL}

\section{Materials and methods}

Thiazolidinones (1) and (2) were prepared by known methods ${ }^{[4]}$ of cyclocondensation of respective ketoanils with thioglycolic acid and purified by recrystallization. Ammine complex $\left.\mathrm{Cu}\left(\mathrm{NH}_{3}\right)_{4}\right] \mathrm{Cl}_{2}$ was prepared and purified by King's methods ${ }^{[5]}$. Metal Chloride and other chemicals (Aldrich, Qualigen and BDH) were used as supplied except solvents which were used after distillation.

\section{Preparation of Complexes}

Complexes resulting from partial replacement of coordinated ammonia by (1) or (2) alone and along with thiourea were prepared by mixing ethanoic solutions of ligands $(0.1 \mathrm{~mol}$ each $)$ with aqueous ethanoic (70\%) solutions of ammine complexes $(0.1 \mathrm{~mol})$ in presence of $\mathrm{NH}_{3}\left(5 \mathrm{~cm}^{3}\right)$. afraction of ligand (1) and thiourea substituted ammine complexes of $\mathrm{Cu}$ (II) precipitated immediately or after refluxing the reaction mixture (4-6hrs) .The products were washed with $\mathrm{EtOH} /$ $\mathrm{Me}_{2} \mathrm{CO}$ and dried in hot air oven at $80^{\circ} \mathrm{C}$; b-fraction isolated from filtrates on concentration and crystallization was washed with $\mathrm{EtOH} / \mathrm{AcOH}$ Toluene(3:1 v/v) $\mathrm{Et}_{2} \mathrm{O}$ and dried at $100^{\circ} \mathrm{C}$ Ligand (II) substituted ammine complexes of $\mathrm{Cu}$ (II) crystallized from their reaction mixtures after their 6-12 hrs refluxing from the reaction mixture containing (2), thiourea and $\left[\mathrm{Cu}(\mathrm{NH} 3)_{4}\right] \mathrm{Cl}_{2}, \mathrm{Cu}_{3}\left(\mathrm{C}_{15} \mathrm{H}_{11} \mathrm{~N}_{2} \mathrm{O}\right.$ S $)$ $\mathrm{Cl}_{6} \cdot 2 \mathrm{H}_{2} \mathrm{O}$ was precipitated immediately whereas $\mathrm{Cu}\left(\mathrm{SCN}_{2} \mathrm{H}_{4}\right)\left(\mathrm{C}_{15} \mathrm{H}_{11} \mathrm{~N}_{2} \mathrm{O}_{3} \mathrm{~S}\right) \mathrm{Cl}_{2} .5 \mathrm{H}_{2} \mathrm{O}$, was isolated on refluxing, concentrating and crystallizing the filterate.

\section{Resolution of binary complexes}

Chromatography was undertaken in a column $(50 \mathrm{~cm}$ length, $2 \mathrm{~cm}$ diameter) containing silica gel (50-100 mesh, BDH) in $\mathrm{AcOH}-\mathrm{C}_{6} \mathrm{H}_{6}(2: 1 \mathrm{v} /$ v). The binary products were dissolved in dimethylsulphoxide and loaded in the column, and the fast moving component eluted. The slow moving component was eluted with an appropriate solvent. Eluates were evaporated to dryness under reduced pressure.

\section{Analysis and Physical Measurements}

$\mathrm{CH}$ and $\mathrm{N}$ analysis were done on Varioel-III Element-R. Melting points determined in open glass capillaries were uncorrected, infrared spectra were recorded on Thermo Nicolet Nexus FT-IR spectrometer in Nujol whereas reflectance spectra were recorded on a Carl-Zeiss VSU-2P spectrophotometer in $\mathrm{MgO}$. Conductometric measurements on standard solutions of complexes in DMSO were made on Toshniwal Conductivity Bridge using a dip-type cell. Magnetic susceptibilities for solids were measured in vibrational magnetometer. Molecular weights were determined by micro Rast's method [6] using Camphor solvent.

\section{RESULTS AND DISCUSSION}

Analysis and molecular weights data are in conformity of proposed molecular formulae. Silver nitrate test revealed non-electrolytic nature of complexes.

IR spectrum of (1) together with ammonia and thiourea exhibits frequencies of $\mathrm{C}-\mathrm{N}$ (cyclic), C-S-C (cyclic) and SH groups at $1600 \mathrm{~cm}^{-1} 690 \mathrm{~cm}^{-1}$ and $2670 \mathrm{~cm}^{-1}$ respectively. Considerable lowering in $\mathrm{C}-\mathrm{N}$ (cyclic) frequency and disappearance of $\mathrm{SH}$ band in complexes led to the inference that (1) is coordinated with metal ions through its thiazolidinone ring nitrogen and deprotonated mercaptyl group. New low frequency peaks corresponding to $\mathrm{M}-\mathrm{N}$ and $\mathrm{M}-\mathrm{S}$ stretches appeared in the spectra of complexes supported the participation of these groups in coordination. Ligand (2) displays $v \mathrm{C}=\mathrm{O}$ (cyclic) and $v \mathrm{C}=\mathrm{N}$ (cyclic) vibrations at $1600 \mathrm{~cm}^{-1}$ and $1590 \mathrm{~cm}^{-1}$ respectively. In the complexes obtained by partial or complete substitution of ammonia by (2) alone or along with $\mathrm{SCN}_{2} \mathrm{H}_{4}$, thiazolidinone ring carbonyl group peak disappeared and pyridine ring $\mathrm{C}=\mathrm{N}$ group frequency lowered considerably. This suggests coordination of thiazolidinone ring carbonyl oxygen after its enolization and pyridine ring nitrogen. Two new low frequency bands corresponding to $\mathrm{M}-\mathrm{O}$ and $\mathrm{M}-\mathrm{N}$ stretches confirm this inference. In the products obtained by partial substitution of ammonia by (1) or (2) an additional band of $v \mathrm{M}-\mathrm{N}$ is observed. If two nitrogens are coordinated one is from thiazolidinone ring or pyridine ring and other should be from ammonia. The low symmetrical deformation (ca. $1608 \mathrm{~cm}^{-1}$ ) and rocking (ca. $842 \mathrm{~cm}^{-1}$ ) vibrations of coordinated ammonia confirm this. In the IR 
Table 1: Magnetic Moment and Reflectance Spectra of Complexes

\begin{tabular}{|c|c|c|c|c|}
\hline \multirow[t]{2}{*}{ Complexes } & \multirow{2}{*}{$\begin{array}{l}\text { Magnetic } \\
\text { Moment } \\
\left(\mu_{\text {eff }}\right) B M \\
\end{array}$} & \multicolumn{2}{|c|}{ Reflectance Spectra } & \multirow[t]{2}{*}{$10 \mathrm{Dq}\left(\mathrm{cm}^{-1}\right)$} \\
\hline & & Band $\left(\mathrm{cm}^{-1}\right)$ & Assignment & \\
\hline $\begin{array}{l}{\left[\mathrm{Cu}\left(\mathrm{NH}_{3}\right)\left(\mathrm{C}_{16} \mathrm{H}_{12} \mathrm{NO}_{3} \mathrm{~S}_{2}\right)\right.} \\
\left.\left(\mathrm{H}_{2} \mathrm{O}\right)_{2} \mathrm{Cl}\right] \cdot 4 \mathrm{H}_{2} \mathrm{O}\end{array}$ & 2.00 & $\left.\begin{array}{l}12563\} \\
15385 \\
23364 \\
24510 \\
25253 \\
26667 \\
27322 \\
40816 \\
47619\end{array}\right\}$ & $\begin{array}{l}{ }^{2} \mathrm{~T}_{2 g} \leftarrow{ }^{2} \mathrm{E}_{\mathrm{g}} \\
{ }^{2} \mathrm{~B}_{2 g} \leftarrow{ }^{2} \mathrm{~B}_{19} \\
{ }^{2} \mathrm{E}_{9}\end{array}$ & 12563 \\
\hline $\begin{array}{l}{\left[\mathrm{Cu}\left(\mathrm{NH}_{3}\right)\left(\mathrm{C}_{16} \mathrm{H}_{12} \mathrm{NO}_{3} \mathrm{~S}_{2}\right)_{2}\right.} \\
\left.\left(\mathrm{H}_{2} \mathrm{O}\right)\right] \cdot \mathrm{H}_{2} \mathrm{O}\end{array}$ & 1.95 & $\begin{array}{l}12626 \\
12920 \\
27548 \\
28409 \\
34483 \\
35336 \\
40162\end{array}$ & $\begin{array}{l}\text { Metal } \leftarrow \text { Ligand } \\
\text { Charge Transfer }\end{array}$ & 12920 \\
\hline $\begin{array}{l}{\left[\mathrm{Cu}\left(\mathrm{NH}_{3}\right)\left(\mathrm{SCN}_{2} \mathrm{H}_{4}\right)\left(\mathrm{C}_{16} \mathrm{H}_{12} \mathrm{NO}_{3} \mathrm{~S}_{2}\right)\right.} \\
\left.\left(\mathrm{H}_{2} \mathrm{O}\right) \mathrm{Cl}\right] . \mathrm{H}_{2} \mathrm{O}\end{array}$ & 1.89 & $\left.\begin{array}{l}12579 \\
13106 \\
21786 \\
23202 \\
23810 \\
27933 \\
35336 \\
37879 \\
40323 \\
43668 \\
48780\end{array}\right\}$ & $\begin{array}{l}{ }^{2} \mathrm{~T}_{2 g} \leftarrow{ }^{2} \mathrm{E}_{9} \\
{ }^{2} \mathrm{~A}_{19} \leftarrow{ }^{2} \mathrm{~B}_{19} \\
{ }^{2} \mathrm{~B}_{2 \mathrm{~g}} \leftarrow \\
{ }^{2} \mathrm{~B}_{9} \leftarrow\end{array}$ & 12579 \\
\hline $\begin{array}{l}{\left[\mathrm{Cu}_{2}\left(\mathrm{NH}_{3}\right)\left(\mathrm{C}_{15} \mathrm{H}_{11} \mathrm{~N}_{2} \mathrm{O}_{3} \mathrm{~S}\right)\right.} \\
\left.\left(\mathrm{H}_{2} \mathrm{O}\right)_{3} \mathrm{Cl}_{4}\right]\end{array}$ & 1.67 & $\left.\begin{array}{l}12920 \\
20964 \\
21552 \\
23529 \\
28248 \\
36232 \\
40816 \\
45455 \\
47170\end{array}\right\}$ & $\begin{array}{l}{ }^{2} \mathrm{~T}_{2 g} \leftarrow^{2} \mathrm{E}_{9} \\
{ }^{2} \mathrm{~A}_{19} \leftarrow^{2} \mathrm{~B}_{19} \\
{ }^{2} \mathrm{~B}_{29} \leftarrow \\
{ }^{2} \mathrm{E}_{g} \leftarrow \\
\text { Metal } \leftarrow \text { Ligand } \\
\text { Charge Transfer }\end{array}$ & 12920 \\
\hline$\left[\mathrm{Cu}_{3}\left(\mathrm{C}_{15} \mathrm{H}_{11} \mathrm{~N}_{2} \mathrm{O}_{3} \mathrm{~S}\right) \mathrm{Cl}_{6}\right] \cdot 2\left(\mathrm{H}_{2} \mathrm{O}\right)$ & 1.70 & $\begin{array}{l}16367^{-} \\
16694 \\
17331 \\
40650 \\
48547\end{array}$ & $\begin{array}{l}{ }^{2} \mathrm{~A}_{19} \leftarrow{ }^{2} \mathrm{~B}_{\mathrm{g}} \\
{ }^{2} \mathrm{~B}_{2 g} \leftarrow \\
{ }^{2} \mathrm{E}_{g} \leftarrow \\
\text { Metal } \leftarrow \text { Ligand } \\
\text { Charge Transfer }\end{array}$ & 12920 \\
\hline $\begin{array}{l}{\left[\mathrm{Cu}\left(\mathrm{SCN}_{2} \mathrm{H}_{4}\right)\left(\mathrm{C}_{15} \mathrm{H}_{11} \mathrm{~N}_{2} \mathrm{O}_{3} \mathrm{~S}\right)\right.} \\
\left.\left(\mathrm{H}_{2} \mathrm{O}\right) \mathrm{Cl}_{2}\right] \cdot 4 \mathrm{H}_{2} \mathrm{O}\end{array}$ & 1.85 & $\left.\begin{array}{l}12594 \\
12920 \\
26455 \\
27174 \\
28090 \\
40323 \\
47170 \\
50000\end{array}\right\}$ & $\begin{array}{l}\text { Metal } \leftarrow \text { Ligand } \\
\text { Charge Transfer }\end{array}$ & \\
\hline
\end{tabular}


spectra of complexes involving (1) or (2), $\mathrm{NH}_{3}$ and $\mathrm{SCN}_{2} \mathrm{H}_{4}$, unperturbed additional ammine frequencies and a new low frequency band (260 $\mathrm{cm}^{-1}$ ) suggest bonding of $\mathrm{SCN}_{2} \mathrm{H}_{4}$ through sulphur. Coordination of monodentate chlorine is indicated by $\mathrm{vM}-\mathrm{Cl}$ band occurring in $300 \mathrm{~cm}^{-1}-320 \mathrm{~cm}^{-1}$ region whereas chlorine bridged polynuclear structures are supported by either a well-defined $v \mathrm{M}-\mathrm{Cl}-\mathrm{M}$ peak or a broad peak arising by mixing of closely spaced $\mathrm{vM}-\mathrm{Cl}$ peak. Lattice water exhibits symmetrical and anti-symmetrical stretching and bonding vibrations in $3321 \mathrm{~cm}^{-1}$ to $3442 \mathrm{~cm}^{-1}$ and $1600 \mathrm{~cm}^{-1}$ to $1625 \mathrm{~cm}^{-1}$ ranges respectively, whereas coordinated water displays $\rho_{\mathrm{t},} \rho_{\mathrm{w}}$ and $\rho_{\mathrm{r}}$ vibrations in $825 \mathrm{~cm}^{-1}-995 \mathrm{~cm}^{-1}$ range. $\mathrm{M}-\mathrm{OH}_{2}$ bands, which generally occur in $200 \mathrm{~cm}^{-1}$ to $450 \mathrm{~cm}^{-1}$ region, could not be clearly identified owing to presence of $v \mathrm{M}$ $\mathrm{N}, v \mathrm{M}-\mathrm{Cl}$ and $v \mathrm{M}-\mathrm{S}$ bands in this region.

\section{Analytical data of complexes}

1. $\left[\mathrm{Cu}\left(\mathrm{NH}_{3}\right)\left(\mathrm{C}_{16} \mathrm{H}_{12} \mathrm{NO}_{3} \mathrm{~S}_{2}\right)\left(\mathrm{H}_{2} \mathrm{O}\right)_{2} \mathrm{Cl}\right] .4 \mathrm{H}_{2} \mathrm{O}$ :

Colour : Grey; M.P:190ㄷ. Anal Calcd.: C,34.65; H, 4.87; N,5.05. Found: C, 34.72; H,4.87; N,5.05. Mol.wt: Calcd: 554; Found:555. IR(cm-1) : vC-N(cyclic), 1500; vC$\mathrm{S}-\mathrm{C}, 702 \mathrm{sh} ; \mathrm{vM}^{-\mathrm{NH}_{3}}$ and/or M-O, 529; vM-N, 457; vM-Cl, 300; vM-S, 270.

2. $\left[\mathrm{Cu}\left(\mathrm{NH}_{3}\right)\left(\mathrm{C}_{16} \mathrm{H}_{12} \mathrm{NO}_{3} \mathrm{~S}_{2}\right)_{2}\left(\mathrm{H}_{2} \mathrm{O}\right)\right] . \mathrm{H}_{2} \mathrm{O}$ :

Colour: Grey; M.P:209${ }^{\circ}$. Anal Calcd.: C,24.72; H, 2.44; N,5.40. Found: C, 24.77; $\mathrm{H}, 2.85$; N,5.40. Mol.wt: Calcd: 777; Found:769. IR( $\left.\mathrm{cm}^{-1}\right): v \mathrm{C}-\mathrm{N}$ (cyclic), 1505; $v \mathrm{C}-$ $\mathrm{S}-\mathrm{C}, 650 ; \mathrm{vM}^{-\mathrm{NH}_{3}}$ and/or M-O, 544; vM-N, 457; vM-S, 290.

3. $\left[\mathrm{Cu}\left(\mathrm{NH}_{3}\right) \quad\left(\mathrm{SCN}_{2} \mathrm{H}_{4}\right) \quad\left(\mathrm{C}_{16} \mathrm{H}_{12} \mathrm{NO}_{3} \mathrm{~S}_{2}\right)(\right.$ $\left.\left.\mathrm{H}_{2} \mathrm{O}\right) \mathrm{Cl}\right] . \mathrm{H}_{2} \mathrm{O}$ :

Colour: Green black; M.P:240ㄷ. Anal Calcd.: C,36.55; H, 4.48; N,10.03. Found: C, 35.99; H,4.33; N,10.15. Mol.wt: Calcd: 558; Found:555. IR( $\left.\mathrm{cm}^{-1}\right): v \mathrm{C}-\mathrm{N}$ (cyclic), 1511; $v \mathrm{C}-$ $\mathrm{S}-\mathrm{C}, 667$; vM-NH3 and/or M-O, 527; vM-N,
433; vM-Cl, 300; vM-S, 285 and 260.

4. $\left[\mathrm{Cu}_{2}\left(\mathrm{NH}_{3}\right)\left(\mathrm{C}_{15} \mathrm{H}_{11} \mathrm{~N}_{2} \mathrm{O}_{3} \mathrm{~S}\right)\left(\mathrm{H}_{2} \mathrm{O}\right){ }_{3} \mathrm{Cl}_{4}\right]$ :

Colour : Brown; M.P : $238^{\circ} \mathrm{C}$. Anal Calcd.: C,30.76; H, 2.29; N,7.17. Found: C, 30.92; H,2.20; N,7.22. Mol.wt: Calcd: 585; Found : 588. IR( $\left.\mathrm{cm}^{-1}\right)$ : vC-N(cyclic), 1625br; vC-SC, 675; vC=N,1433; vM-NH3 and/or M-O, 500 and 467br; vM-N, 417, 467br; vM-Cl, 300 and 280.

5. $\quad\left[\mathrm{Cu}_{3}\left(\mathrm{C}_{15} \mathrm{H}_{11} \mathrm{~N}_{2} \mathrm{O}_{3} \mathrm{~S}\right) \mathrm{Cl}_{6}\right] \cdot 2\left(\mathrm{H}_{2} \mathrm{O}\right)$ :

Colour: Grey; M.P : $>300^{\circ} \mathrm{C}$. Anal Calcd: C,24.37; H, 2.03; N,3.79. Found: C, 24.05; H,2.44; N,3.83. Mol.wt: Calcd: 775; Found : 769. IR $\left(\mathrm{cm}^{-1}\right)$ : vC-S-C, 674; vC $=\mathrm{N}, 1449 ; \mathrm{vM}-$ $\mathrm{NH}_{3}$ and/or M-O, 483br; vM-N, 434; vM-Cl, 295br.

6. $\left[\mathrm{Cu}\left(\mathrm{SCN}_{2} \mathrm{H}_{4}\right)\left(\mathrm{C}_{15} \mathrm{H}_{11} \mathrm{~N}_{2} \mathrm{O}_{3} \mathrm{~S}\right)\left(\mathrm{H}_{2} \mathrm{O}\right) \mathrm{Cl}_{2}\right] \cdot 4 \mathrm{H}_{2} \mathrm{O}$ Colour : brown; M.P:200ㄷ. Anal Calcd.: $\mathrm{C}, 32.02 ; \mathrm{H}, 4.17$; N,9.34. Found: C, 31.62; $\mathrm{H}, 4.27$; N,9.28. Mol.wt: Calcd: 599; Found:588. IR( $\left.\mathrm{cm}^{-1}\right)$ : vC-N(cyclic), 1608; $v \mathrm{C}=\mathrm{N}, 1466 ; \mathrm{vM}-\mathrm{NH} 3$ and/or $\mathrm{M}-\mathrm{O}, 500$ and 475br; vM-N, 417; vM-CI, 305; vM-S, 260.

Band frequencies, assignments and values of ligand field parameters obtained by standard treatment ${ }^{8}$ are in table 1.

The magnetic moment and reflectance spectrum of $\left[\mathrm{Cu}_{3}\left(\mathrm{C}_{15} \mathrm{H}_{11} \mathrm{~N}_{2} \mathrm{O}_{3} \mathrm{~S}\right)\right] \cdot 2 \mathrm{H}_{2} \mathrm{O}$ displaying three $\mathrm{d}$ - $d$ transition bands in $16367-17331 \mathrm{~cm}^{-1}$ range, characteristic of $D_{4 h}$ symmetry ${ }^{1,9}$, indicate its square-planar geometry. All other copper (II) complexes displaying one or two broad bands in $12516-15385 \mathrm{~cm}^{-1}$ range and other two or three bands in $16367-24510 \mathrm{~cm}^{-1}$ region indicate ${ }^{[10]}$ their distorted octahedral stereochemistry involving ${ }^{2}$ $\mathrm{T}_{2 g} \leftarrow{ }^{2} \mathrm{E}_{\mathrm{g}}$ transitions; the ${ }^{2} \mathrm{E}_{\mathrm{g}}$ state being susceptible to John-Teller distortion may account for the broad peak structure.

\section{REFERENCES}

1. R.K.Upadhyay, A.K. Bajpai, and K. Rathore, 4. Transition Met. Chem, 1985; 10, 24.

2. R.K.Upadhyay, J. Indian Chem.Soc, 1997; 74, 214.

3. R.K.Upadhyay, J. Indian Chem.Soc, 1997, $75,535$.
4. R.K.Upadhyay, N.K.Agarwal and N.Gupta, J. Indian Chem.Soc., 1993, 70, 537.

5.. A.King in A.J.E. Welch(Ed.), Inorganic Preparation, Allen and Unwin, London, 1950. S.Glasstone, A Text Book of Physical Chemistry, Macmillan and co. London, 1974 
7. R.K.Upadhyay, D.Sc.Thesis, C.C.S University, Merrut, 1994.

8. E.Konig, Structure and Bonding, 1971, 9, 175.

9. N.M. Karayaumis, C.M.Milkulski, I.L.
Pytlewski and M.M.Labes, J. Inorganic nucl Chem.,1972, 34, 3139.

10. B.Singh, P.L.Maurya, B.V.Agarwala and A.K.Dey, J. Indian Chem..Soc, 1981, 58, 541. 